J. Kuhl • A. Hilding $\cdot$ C. G. Östenson • V. Grill •

S. Efendic $\cdot$ P. Båvenholm

\title{
Characterisation of subjects with early abnormalities of glucose tolerance in the Stockholm Diabetes Prevention Programme: the impact of sex and type 2 diabetes heredity
}

Received: 27 June 2004 / Accepted: 15 September 2004 / Published online: 24 December 2004

C) Springer-Verlag 2004

\begin{abstract}
Aims/hypothesis: We evaluated the impact of sex and type 2 diabetes heredity on the prevalence and pathogenesis of early abnormalities of glucose homeostasis in subjects participating in the Stockholm Diabetes Prevention Programme. Methods: A sample of 3,128 men and 4,821 women, of whom approximately half had a family history of type 2 diabetes (FHD) was categorised according to an OGTT: NGT, IFG, IGT, combined glucose intolerance and type 2 diabetes. The homeostasis model assessment was used to determine insulin sensitivity and beta cell function. Results: Prevalence of early abnormalities of glucose metabolism was two to three times higher in subjects with FHD and two to three times higher in men compared to women. Both maternal and paternal heredity of type 2 diabetes were associated with an increased risk of having early abnormalities of glucose metabolism. However, in women with type 2 diabetes heredity on the father's side seems to have less impact on an increased risk of having type 2 diabetes. Both waist circumference and systolic blood pressure were increased in subjects with abnormalities of glucose homeostasis, whereas insulin sensitivity and beta cell function were decreased. Subjects with IFG had more pronounced impairment of beta cell function and insulin sensitivity than subjects with IGT. Conclusion/interpretation: An FHD and male sex increased the prevalence of abnormalities of glucose homeostasis. Subjects with IFG had more pronounced defects of insulin secretion and action than subjects with IGT.
\end{abstract}

Keywords Glucose intolerance · Insulin secretion · Insulin sensitivity · Type 2 diabetes heredity

\footnotetext{
J. Kuhl $(\bowtie) \cdot$ A. Hilding · C. G. Östenson · V. Grill · S. Efendic Department of Molecular Medicine, Karolinska Institute, 17176 Stockholm, Sweden

e-mail: jeanette.kuhl@molmed.ki.se

Tel.: +46-8-51772535

Fax: $+46-8-51773968$

P. Båvenholm

Department of Medicine, Karolinska Hospital,

Stockholm, Sweden
}

Abbreviations CGI: combined glucose intolerance . CVD: cardiovascular disease - FHD: family history of type 2 diabetes - HOMA: homeostasis model assessment - PI: proinsulin - POR: prevalence odds ratios - SDPP: Stockholm Diabetes Prevention Program - WHO: World Health Organization

\section{Introduction}

Type 2 diabetes develops slowly through stages of early impairments of glucose metabolism. The presence of IFG or IGT carries a similar risk of developing type 2 diabetes, although the highest incidence is among those who have combined glucose intolerance (CGI). Thus, a majority of subjects who develop type 2 diabetes demonstrate IFG and/or IGT approximately 5 years prior to onset of the disease [1]. Heredity plays an important role in the pathogenesis of type 2 diabetes [2]. Less is known about the impact of heredity on the development of early abnormalities of glucose metabolism, such as IFG, IGT and CGI. Furthermore, it is not known whether there is a difference between the impact of maternal or paternal diabetes heredity, in males and females, on development of glucose abnormalities.

In most populations, IGT is more prevalent than IFG and therefore more sensitive (not specific) for the purpose of identifying individuals at risk of developing type 2 diabetes. However, several large-scale studies report poor agreement between the two features of disturbed glucose homeostasis [3, 4], suggesting different pathogenic backgrounds. Two studies report that subjects with IFG exhibit more pronounced insulin resistance compared to IGT, while subjects with IGT display deficits in early and late phase insulin secretion $[5,6]$. In another study, subjects with IFG were both more insulin resistant and had poorer beta cell function compared to IGT subjects [7]. On the contrary, one study showed that insulin resistance was absent in IFG and markedly pronounced in IGT, whereas a defective insulin response was significant only in IFG [8]. 
To further explore the impact of sex and type 2 diabetes heredity on the prevalence and pathogenesis of early abnormalities of glucose metabolism we have studied 7,949 healthy men and women, of whom, by design, approximately half had a family history of type 2 diabetes (FHD). Participants were investigated between 1992 and 1998 and belonged to a cross-sectional study of Swedish Caucasians born between 1938 and 1961, the Stockholm Diabetes Prevention Programme (SDPP) [9-11]. An OGTT was performed, and blood pressure levels and anthropometrical data were recorded. Insulin sensitivity and beta cell function were assessed using the homeostasis model assessment (HOMA) indices. In addition, fasting plasma proinsulin (PI) levels were measured in the male cohort.

\section{Subjects and methods}

Subjects All participants belonged to the SDPP, described in detail elsewhere [9-12]. In short, the study consisted of men and women, aged 35-56 years, with or without FHD, and without previously diagnosed type 2 diabetes. The subjects were living in five municipalities situated in the outskirts of Stockholm.

The data collection was performed in two steps. First, a questionnaire was sent to all inhabitants in the relevant age group asking about the subject's country of birth and presence of type 2 diabetes in the family as well as in the subject. The questionnaire was returned by $79 \%(10,236$ of 12,952$)$ of the men and $85 \%(16,481$ of 19,416$)$ of the women. Subjects who had known type 2 diabetes, who were of foreign origin, who provided incomplete answers, or presented insufficient FHD, were excluded from further investigations.

Secondly, all subjects with FHD, defined as at least one first-degree relative (mother, father, sister or brother) or two second-degree relatives (grandparents, uncles or aunts) with diagnosed type 2 diabetes $(2,106$ men and 3,583 women) were invited to participate in a health examination at a primary health care centre. Of the responders, $20 \%$ of the men and $24 \%$ of the women had FHD. An age-stratified sample of subjects without FHD, in total 2,424 men and 3,497 women (together with 424 women reporting gestational diabetes), were also invited to participate in the examination. In total, 3,162 (70\%) men and 4,946 (66\%) women agreed to participate in the examination. Due to uncertain heredity and because of women who were also pregnant or breast-feeding and for medical reasons, some subjects were excluded at this stage. Thus, the final SDPP sample consisted of 3,128 men and 4,821 women. All participants received written and oral information regarding the study and gave their informed consent. The experimental protocol was approved by the Ethics Committee at the Karolinska Hospital and was carried out in accordance with the Declaration of Helsinki.

Study protocol During the visit to the primary health care centre, the subjects filled out an extensive questionnaire that included questions on birthweight, physical activity, dietary habits, health status, alcohol and tobacco use and psychosocial conditions. Body height, weight and waist circumference were measured with the subjects wearing light indoor clothes and no shoes. BMI was calculated as the ratio of body weight in kilograms to the square of height in metres $\left(\mathrm{kg} / \mathrm{m}^{2}\right)$. A standard 75-g OGTT, according to World Health Organization (WHO) criteria [13], was administered to the subjects. According to the ADA document from 1997 [14] the criteria for IFG were a fasting plasma glucose level of $\geq 6.1$ to $<7.0 \mathrm{mmol} / 1$ and a 2-h plasma glucose level at OGTT $<7.8 \mathrm{mmol} / \mathrm{l}$; for IGT a fasting plasma glucose level $<6.1 \mathrm{mmol} / \mathrm{l}$ and a $2-\mathrm{h}$ plasma glucose level at OGTT of $\geq 7.8$ to $<11.1 \mathrm{mmol} / \mathrm{l}$; and for CGI a fasting plasma glucose level $\geq 6.1$ and $<7.0$ $\mathrm{mmol} / \mathrm{l}$ and 2-h plasma glucose level at OGTT $\geq 7.8$ and $<11.1 \mathrm{mmol} / \mathrm{l}$. Type 2 diabetes was defined as having a fasting plasma glucose value $\geq 7.0 \mathrm{mmol} / \mathrm{l}$ and/or a 2 -h plasma glucose value at OGTT $\geq 11.1 \mathrm{mmol} / \mathrm{l}$.

The HOMA was used to assess insulin sensitivity and beta cell function, based on fasting insulin and glucose levels and according to published algorithms: HOMA resistance $=($ insulin $\times$ glucose $) / 22.5$, and HOMA beta cell function $=20 \times$ insulin $/($ glucose -3.5$)$ [15].

Statistical analysis Data are presented as means \pm SEM or geometric mean (SE range). Plasma glucose, plasma insulin, HOMA values and plasma PI were log-transformed to improve skewness. Analysis of variance was used, and when controlling for covariates, analysis of covariance, both followed by Scheffe's post-hoc test, was performed to analyse differences in continuous variables between groups. Systolic and diastolic blood pressure, fasting and 2-h insulin values, HOMA measures, PI and PI insulin ratios are presented after adjustment for age, FHD, waist circumference, sex and when appropriate also for current smoking, physical activity at work and during leisure time. When a significant interaction term between glucose tolerance groups and age or waist circumference was found results are presented with age or waist circumference at their means. Multiple logistic regressions were performed to calculate prevalence odds ratios (POR), accompanied by $95 \% \mathrm{CI}$. Including them one by one in the logistic regression model tested for potential confounders. They were retained in the model if they contributed to at least a 10\% change of the age-adjusted (age in three categories 35-42, 43-50, 51-56 years) crude estimate. Statistical significance was set at $p<0.05$. Data processing was performed using the Statistical Analysis System, SAS, version 8.2 (Cary, NC, USA), and Statistica, StatSoft, version 6.1 (Tulsa, OK, USA).

Assays Plasma glucose was assayed in duplicate using a glucose oxidase method with a Yellow Spring Glucose Analyzer (Yellow Springs, Inc., Yellow Springs, OH, USA). Insulin was assayed by RIA, using our own antibodies, human insulin as a standard, and charcoal addition to separate antibody-bound and free insulin [16]. Proinsulin cross-reacts in this assay by about $80 \%$. Pro- 
Table 1 Glucose tolerance in subjects with or without heredity for type 2 diabetes

\begin{tabular}{llrrrrr}
\hline & Sex & \multicolumn{2}{c}{ FHD positive } & & \multicolumn{2}{c}{ FHD negative } \\
\cline { 3 - 3 } \cline { 6 - 6 } & & Number & Percent & & Number & Percent \\
\hline \multirow{2}{*}{ NGT } & Male & 1,422 & 87.7 & & 1,413 & 93.8 \\
& Female & 2,398 & 92.8 & 2,152 & 96.2 \\
IFG & Male & 38 & 2.3 & 21 & 1.4 \\
& Female & 33 & 1.3 & 15 & 0.7 \\
IGT & Male & 91 & 5.6 & 52 & 3.5 \\
& Female & 95 & 3.7 & 50 & 2.2 \\
CGI & Male & 19 & 1.2 & 7 & 0.5 \\
& Female & 12 & 0.5 & 3 & 0.1 \\
Type 2 diabetes & Male & 51 & 3.1 & 14 & 0.9 \\
& Female & 45 & 1.7 & 18 & 0.8 \\
Total & Male & 1,621 & 100.0 & 1,507 & 100.0 \\
& Female & 2,583 & 100.0 & 2,238 & 100.0 \\
\hline
\end{tabular}

CGI Combined glucose intolerance, FHD positive with family history of type 2 diabetes, $F H D$ negative without family history of type 2 diabetes

insulin was determined in men by an ELISA with a broad specificity comprising, in addition to intact PI, the four PI conversion intermediates, reacting with $65-99 \%$ efficiency relative to intact PI. The limit of detection was $0.25 \mathrm{pmol} /$ 1 , and the total interassay coefficient of variation was 7.6$5.3 \%$ at $1.1-8 \mathrm{pmol} / 1$.

\section{Results}

Influence of sex and type 2 diabetes heredity on prevalence of glucose abnormalities In total, 2,835 men (90.6\%) and 4,550 women $(94.4 \%)$ had NGT. The corresponding figures for IFG were 59 men $(1.9 \%)$ and 48 women $(1.0 \%)$, and for IGT 143 men $(4.6 \%)$ and 145 women (3.0\%), and for CGI 26 men $(0.8 \%)$ and 15 women $(0.3 \%)$, and finally 65 men $(2.1 \%)$ and 63 women (1.3\%) had type 2 diabetes. Hence, the prevalence of having glucose intolerance, which includes IFG, IGT and diabetes, was ap- proximately twofold higher in men compared to women. There was an overlap between IFG and IGT, 31\% of the male subjects with IFG had IGT, whereas only $15 \%$ of male subjects with IGT also had IFG. The corresponding figures for women were 24 and $9 \%$, respectively. Table 1 shows the distribution of men and women in all glucose tolerance categories when grouped according to FHD. The prevalence of having type 2 diabetes or milder forms of disturbed glucose metabolism was approximately two to three times higher in subjects with FHD. In total, $12.3 \%$ of men and $7.2 \%$ of women with FHD had an abnormal glucose metabolism, whereas the corresponding figure for men without FHD was $6.2 \%$ and for women $3.8 \%$. Interestingly, prevalence of type 2 diabetes was similar in both sexes in subjects without FHD, but more than threefold increased in men with FHD compared to a twofold increase in women.

Impact of parental type 2 diabetes heredity on the prevalence of glucose abnormalities In both men and women, the POR for having early abnormalities of glucose metabolism were similar in those who had maternal and paternal diabetes heredity (Table 2). In women, diabetes heredity on the mother's side, but not on the father's side, was associated with an increased risk of having type 2 diabetes. In men, however, both maternal and paternal diabetes heredity were associated with type 2 diabetes.

Clinical characteristics Basic characteristics of the study population are given in Table 3. Individuals with IFG, IGT and type 2 diabetes were slightly older than those with NGT $(p<0.05$ to $p<0.001)$. There was no difference in birthweight between the groups. In both males and females there was significant increase in BMI and waist circumference in subjects with IFG, IGT, CGI and type 2 diabetes $(p<0.01$ to $p<0.001)$. Systolic blood pressure was higher in subjects with IFG, IGT, CGI and type 2 diabetes compared to NGT ( $p<0.01$ to $p<0.001$ ). Diastolic blood pressure was higher in subjects with IGT compared to NGT $(p<0.001)$.

Insulin levels, insulin sensitivity and beta cell function Both fasting and 2-h plasma insulin levels were higher in

Table 2 Prevalence odds ratio (POR) of glucose intolerance associated with family history of type 2 diabetes on the father's or mother's side

\begin{tabular}{|c|c|c|c|c|c|c|c|}
\hline \multirow[t]{2}{*}{ FHD } & \multirow[t]{2}{*}{ NGT $(n)$} & \multicolumn{3}{|c|}{ 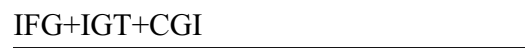 } & \multicolumn{3}{|c|}{ Type 2 diabetes } \\
\hline & & Number & POR & $95 \% \mathrm{CI}$ & Number & POR & $95 \% \mathrm{CI}$ \\
\hline \multicolumn{8}{|l|}{ Men } \\
\hline No & 1,409 & 80 & 1.0 & & 14 & 1.0 & \\
\hline Father's side & 508 & 51 & 1.6 & $1.1-2.4$ & 15 & 2.8 & $1.3-5.8$ \\
\hline Mother's side & 536 & 61 & 1.8 & $1.2-2.5$ & 22 & 3.6 & $1.8-7.0$ \\
\hline \multicolumn{8}{|l|}{ Women } \\
\hline No & 2,147 & 67 & 1.0 & & 18 & 1.0 & \\
\hline Father's side & 800 & 44 & 1.5 & $1.0-2.3$ & 10 & 1.3 & $0.6-2.8$ \\
\hline Mother's side & 940 & 50 & 1.4 & $1.0-2.1$ & 20 & 2.0 & $1.1-3.9$ \\
\hline
\end{tabular}

Adjusted for age $(35-42,43-50,51-56$ years $)$ and BMI $(<25.0,25.0-29.9, \geq 30.0)$

$C G I$ Combined glucose intolerance, $F H D$ family history of type 2 diabetes 
Table 3 Basic characteristics of the study population (3,128 men and 4,821 women)

\begin{tabular}{|c|c|c|c|c|c|}
\hline & \multicolumn{5}{|c|}{ Glucose tolerance group } \\
\hline & NGT & IFG & IGT & CGI & Type 2 diabetes \\
\hline No. & 7,385 & 107 & 288 & 41 & 128 \\
\hline Male/female & $2,835 / 4,550$ & $59 / 48$ & $143 / 145$ & $26 / 15$ & $65 / 63$ \\
\hline Age (years) & $46.8 \pm 0.1$ & $48.7 \pm 0.5^{\mathrm{b}}$ & $47.9 \pm 0.3^{\mathrm{a}}$ & $47.3 \pm 0.8$ & $49.2 \pm 0.4^{\mathrm{c}}$ \\
\hline Birthweight (g) & $3,471 \pm 7$ & $3,359 \pm 64$ & $3,370 \pm 40$ & $3,379 \pm 114$ & $3,342 \pm 59$ \\
\hline \multicolumn{6}{|l|}{ BMI $\left(\mathrm{kg} / \mathrm{m}^{2}\right)$} \\
\hline Male & $25.8 \pm 0.1$ & $27.6 \pm 0.4^{\mathrm{b}}$ & $29.1 \pm 0.3^{\mathrm{c}}$ & $29.3 \pm 0.7^{\mathrm{c}}$ & $29.3 \pm 0.4^{\mathrm{c}}$ \\
\hline Female & $25.2 \pm 0.1$ & $28.6 \pm 0.6^{\mathrm{c}}$ & $28.6 \pm 0.3^{\mathrm{c}}$ & $36.6 \pm 1.1^{\mathrm{c}, \mathrm{g}, \mathrm{f}}$ & $30.7 \pm 0.5^{\mathrm{c}, \mathrm{d}, \mathrm{i}}$ \\
\hline \multicolumn{6}{|l|}{ Waist (cm) } \\
\hline Male & $91.8 \pm 0.2$ & $97.6 \pm 1.1^{\mathrm{c}}$ & $99.4 \pm 0.7^{\mathrm{c}}$ & $100.5 \pm 1.6^{\mathrm{c}}$ & $100.2 \pm 1.0^{\mathrm{c}}$ \\
\hline Female & $79.9 \pm 0.1$ & $88.8 \pm 1.4^{\mathrm{c}}$ & $88.0 \pm 0.8^{\mathrm{c}}$ & $105.7 \pm 2.5^{\mathrm{c}, \mathrm{g}, \mathrm{f}}$ & $93.7 \pm 1.2^{\mathrm{c}, \mathrm{e}, \mathrm{h}}$ \\
\hline Systolic blood pressure $(\mathrm{mmHg})^{\mathrm{j}}$ & $122.4 \pm 0.2$ & $131.2 \pm 1.5^{\mathrm{c}}$ & $128.4 \pm 0.9^{\mathrm{c}}$ & $133.3 \pm 2.6^{\mathrm{b}}$ & $128.2 \pm 1.3^{\mathrm{c}}$ \\
\hline Diastolic blood pressure $(\mathrm{mmHg})^{\mathrm{j}}$ & $76.9 \pm 0.1$ & $79.8 \pm 1.1$ & $81.1 \pm 0.6^{\mathrm{c}}$ & $74.8 \pm 2.5$ & $79.3 \pm 1.1$ \\
\hline
\end{tabular}

Values are means $\pm \mathrm{SE}$

CGI Combined glucose tolerance

ANOVA or analysis of covariance followed by Scheffé's test vs NGT, ${ }^{\mathrm{a}} p<0.05,{ }^{\mathrm{b}} p<0.01,{ }^{\mathrm{c}} p<0.001 ;$ vs IGT, ${ }^{\mathrm{d}} p<0.05,{ }^{\mathrm{e}} p<0.01,{ }^{\mathrm{f}} p<0.001 ; \mathrm{vs}$ IFG, ${ }^{\mathrm{g}} p<0.001 ;$ vs CGI, ${ }^{\mathrm{h}} p<0.01,{ }^{\mathrm{i}} p<0.001$

${ }^{\mathrm{j}}$ Values after adjustment for age, waist, FHD, smoking, sex

Table 4 Glucose and insulin levels, insulin sensitivity and beta cell function (3,128 men and 4,821 women)

\begin{tabular}{|c|c|c|c|c|c|}
\hline & \multicolumn{5}{|c|}{ Glucose tolerance group } \\
\hline & NGT & IFG & IGT & CGI & Type 2 diabetes \\
\hline No. & 7,385 & 107 & 288 & 41 & 128 \\
\hline Male/female & $2,835 / 4,550$ & $59 / 48$ & $143 / 145$ & $26 / 15$ & $65 / 63$ \\
\hline Fasting P-glucose $(\mathrm{mmol} / \mathrm{l})$ & $4.63(4.63-4.64)$ & $6.35(6.29-6.42)^{\mathrm{c}}$ & $5.11(5.08-5.14)^{\mathrm{c}, \mathrm{i}}$ & $6.42(6.31-6.53)^{\mathrm{c}, \mathrm{f}}$ & $7.38(7.31-7.45)^{\mathrm{c}, \mathrm{i}, \mathrm{f}, \mathrm{j}}$ \\
\hline 2-h P-glucose (mmol/l) & $4.41(4.39-4.42)$ & $5.90(5.76-6.05)^{\mathrm{c}}$ & $8.75(8.61-8.88)^{\mathrm{c}, \mathrm{i}}$ & $9.05(8.69-9.43)^{\mathrm{c}, \mathrm{i}}$ & $12.43(12.14-12.72)^{\mathrm{c}, \mathrm{i}, \mathrm{f}, \mathrm{j}}$ \\
\hline Fasting P-insulin $(\mathrm{pmol} / \mathrm{l})^{\mathrm{k}}$ & $76(76-77)$ & $98(93-103)^{\mathrm{c}}$ & $86(84-89)^{\mathrm{c}}$ & $109(98-121)^{\mathrm{a}}$ & $96(92-101)^{\mathrm{c}}$ \\
\hline 2-h P-insulin $(\mathrm{pmol} / \mathrm{l})^{\mathrm{k}}$ & $231(229-232)$ & $330(312-348)^{\mathrm{c}}$ & $469(453-485)^{\mathrm{c}, \mathrm{i}}$ & $364(331-401)^{\mathrm{c}}$ & $332(316-350)^{\mathrm{c}, \mathrm{f}}$ \\
\hline Fasting P-PI (pmol/ll) & $6.8(6.7-6.8)$ & $13.7(12.7-14.7)^{\mathrm{c}}$ & $9.8(9.4-10.3)^{\mathrm{c}, \mathrm{h}}$ & $15.8(14.2-17.6)^{\mathrm{c}, \mathrm{e}}$ & $18.3(17.0-19.6)^{\mathrm{c}, \mathrm{f}}$ \\
\hline Fasting PI : IRI ratio $(\%)^{1}$ & $6.2(6.1-6.2)$ & $9.9(9.3-10.5)^{\mathrm{c}}$ & $7.7(7.4-8.0)^{\mathrm{c}, \mathrm{g}}$ & $10.7(9.7-11.7)^{\mathrm{c}, \mathrm{d}}$ & $11.2(10.6-11.9)^{\mathrm{c}, \mathrm{f}}$ \\
\hline HOMA-IR ${ }^{\mathrm{k}}$ & $2.62(2.61-2.64)$ & $4.57(4.34-4.81)^{\mathrm{c}}$ & $3.20(3.10-3.29)^{\mathrm{c}, \mathrm{i}}$ & $5.30(4.74-5.93)^{\mathrm{c}, \mathrm{f}}$ & $5.21(4.94-5.49)^{\mathrm{c}, \mathrm{f}}$ \\
\hline HOMA beta cell ${ }^{\mathrm{k}}$ & $236(235-238)$ & $123(117-131)^{\mathrm{c}}$ & $208(201-215)^{\mathrm{a}, \mathrm{i}}$ & $133(122-146)^{\mathrm{c}, \mathrm{f}}$ & $113(107-119)^{\mathrm{c}, \mathrm{f}}$ \\
\hline
\end{tabular}

Values are geometrical mean (SE range)

CGI Combined glucose intolerance, HOMA-IR homeostasis model assessment of insulin resistance, IRI immunoreactive insulin, $P$ plasma, PI proinsulin

ANOVA or analysis of covariance followed by Scheffé's test vs NGT, ${ }^{\mathrm{a}} p<0.05,{ }^{\mathrm{b}} p<0.01,{ }^{\mathrm{c}} p<0.001 ;$ vs IGT, ${ }^{\mathrm{d}} p<0.05,{ }^{\mathrm{e}} p<0.01,{ }^{\mathrm{f}} p<0.001 ; \mathrm{vs}$ IFG, ${ }^{\mathrm{g}} p<0.05,{ }^{\mathrm{h}} p<0.01,{ }^{\mathrm{i}} p<0.001 ; \mathrm{vs}$ CGI, ${ }^{\mathrm{j}} p<0.001$

${ }^{\mathrm{k}}$ Values after adjustment for age, waist, family history of diabetes (FHD), smoking, sex, physical activity

${ }^{\mathrm{l}}$ Values after adjustment for age, waist, FHD, physical activity during leisure time and only measured in men

IFG, IGT, CGI and type 2 diabetes compared to NGT $(p<0.05$ to $p<0.001)$ (Table 4$)$. The HOMA model revealed that both beta cell function and insulin sensitivity were decreased in IFG, IGT, CGI and type 2 diabetes, as compared to NGT. However, subjects with IFG and CGI had more impaired beta cell function compared to IGT $(p<0.001)$, and similarly subjects with IFG and CGI had decreased insulin sensitivity compared to IGT $(p<0.001)$.

Proinsulin concentrations Fasting plasma PI levels were significantly higher in men with IFG, IGT, CGI and type 2 diabetes compared to NGT $(p<0.001)$ (Table 4$)$. Interestingly, subjects with IFG and CGI had significantly higher
PI levels and PI : insulin ratios compared to IGT $(p<0.05$ to $p<0.01)$.

\section{Discussion}

The SDPP study is a large cross-sectional study that included almost 8,000 healthy Swedish men and women who underwent an OGTT. By design, approximately 50\% of included subjects had type 2 diabetes heredity. In this study, we demonstrate for the first time that prevalence of IFG, similarly to prevalence of IGT, CGI and type 2 diabetes, was nearly twofold higher in those who had FHD 
compared to those without FHD. Moreover, prevalence of milder forms of disturbed glucose metabolism was higher in men than in women, irrespective of type 2 diabetes heredity. This contradicts what has been found in other European studies, which demonstrated higher prevalence of IGT in women irrespective of age [17]. Our finding could not, however, be accounted for by differences in ethnicity between men and women since all participants had a Swedish Caucasian background. Of note, prevalence of type 2 diabetes was similar in both sexes in subjects without type 2 diabetes heredity. The present study also shows that maternal and paternal heredity of type 2 diabetes similarly influenced the risk of having early abnormalities of glucose metabolism in both sexes. However, the POR of having type 2 diabetes was higher in men than in women. In fact, in women only diabetes heredity on the mother's side influenced the risk of having type 2 diabetes. Recently, Groop et al. [18] have investigated the impact of parental diabetes on metabolic parameters and phenotype in offspring, and suggested a possibility of unprecedented maternal or paternal inheritance of different type 2 diabetes phenotypes.

There was a poor concordance between the diagnosis of IFG and IGT, in concert with what has been reported by others [3, 4]. In fact, $85 \%$ of the male and $91 \%$ of females with IGT, irrespective of FHD, had normal fasting glucose levels in our study, but slightly lower figures have been reported by others [6-8]. Furthermore, only $31 \%$ of the men and $24 \%$ of the women with IFG also had IGT, a frequency similar to that reported in some studies $[6,8]$, but lower than that found by others $[5,7]$. Our study is the only one that includes, as a separate category, subjects without FHD. Importantly, all studies consistently show that the majority of subjects with IGT will be overlooked if the fasting plasma glucose concentration alone is considered. This demonstrates that IFG is no substitute for OGTT in the identification of subjects at risk of developing type 2 diabetes.

Previous cross-sectional and cohort studies suggest that pronounced insulin resistance is a determinant of IFG in subjects with FHD, whereas both moderate insulin resistance and impaired beta cell function contribute to IGT [6, 7]. In our study, insulin resistance, as assessed by fasting plasma insulin levels and the HOMA insulin resistance index, is a striking feature in both men and women with IFG and IGT. The HOMA model has its obvious limitations, since it measures insulin sensitivity and beta cell function using only fasting values of glucose and insulin. However, the HOMA model has been validated against euglycaemic, hyperinsulinaemic clamps, fasting insulin concentration and hyperglycaemic clamps [15]. Interestingly, the HOMA analysis showed that subjects with IFG have more pronounced impairments of beta cell function than subjects with IGT. We also observed increased PI levels in males with IFG as compared to IGT, which may indicate more pronounced impaired beta cell responsiveness in IFG [19]. However, an influence of insulin resistance in this context cannot be ruled out [20]. Prospective studies show that PI, independently of intact insulin, predicts CHD in subjects with or without type 2 diabetes [21-23]. Proinsulin may be an important marker of a dysmetabolic syndrome $[20,24]$. In the present study, hyperproinsulinaemia in men with early abnormalities of glucose tolerance may partially account for a well-documented increased cardiovascular disease (CVD) risk in these groups [1].

Fasting glucose concentrations primarily depend on hepatic glucose production [25], whereas both hepatic and extra-hepatic insulin sensitivity, and insulin secretion are important determinants of glucose tolerance [26]. In view of these findings it is difficult to understand that subjects with IFG maintained normal OGTT, despite a marked impairment of insulin sensitivity and beta cell function. The present study design did not allow direct measurements of hepatic or extra-hepatic insulin sensitivity [27], nor were gut hormones, alpha cell responsiveness or glucose effectiveness measured, factors that may be important for determining normal glucose pattern during OGTT in IFG [28, 29].

The diastolic and systolic blood pressures, BMI and waist circumference were higher in subjects with abnormalities of glucose metabolism compared to NGT, but birthweight was similar. Also the GENNID study reported higher blood pressure in IFG and/or IGT compared to NGT [7]. It is reasonable to assume that an increase in CVD risk among IFG, and/or IGT [1], is partly mediated by an increase in blood pressure.

It is important to note that the cross-sectional design allows us to assess prevalence and associations of various parameters characterising phenotype of subjects studied. However, the significance of the findings in the SDPP study are limited due to selection bias and therefore not representative of the entire population.

In conclusion, FHD increases the prevalence of IFG, IGT, CGI and type 2 diabetes by two- to threefold in middle-aged men and women. Abnormalities of glucose metabolism were approximately twofold more common in men than women irrespective of type 2 diabetes heredity. Both maternal and paternal heredity of type 2 diabetes influenced the risk of having early abnormalities of glucose metabolism in both sexes, although the parental impact on having type 2 diabetes was higher in men than in women. An OGTT is mandatory for identification of subjects with a risk of developing type 2 diabetes. IFG and IGT subjects exhibited higher blood pressure, increased central fat and decreased insulin action and insulin secretion. IFG had more pronounced defects of both insulin secretion and action compared to IGT, suggesting different pathogenic backgrounds to the two conditions.

Acknowledgements This study was supported by grants from the Swedish Medical Research Council, the Stockholm County Council, and the Swedish Council for Working Life and Social Research, Vårdalstiftelsen and Glaxo Smithkline, Sweden. We thank the nurses and other staff members at the health care centres who carried out the OGTTs and other measures. 


\section{References}

1. Unwin N, Shaw J, Zimmet P, Alberti KG (2002) Impaired glucose tolerance and impaired fasting glycaemia: the current status on definition and intervention. Diabet Med 19:708-723

2. Köbberling J, Tillil H (1982) Empirical risk figures for firstdegree relatives of non-insulin dependent diabetics. In: Köbberling J, Tattersall R (eds) The genetics of diabetes mellitus. Academic Press, London, pp 201-209

3. de Vegt F, Dekker JM, Stehouwer CD, Nijpels G, Bouter LM, Heine RJ (1998) The 1997 American Diabetes Association criteria versus the 1985 World Health Organization criteria for the diagnosis of abnormal glucose tolerance: poor agreement in the Hoorn Study. Diabetes Care 21:1686-1690

4. DECODE (1999) Glucose tolerance and mortality: comparison of WHO and American Diabetes Association diagnostic criteria. The DECODE study group. European Diabetes Epidemiology Group. Diabetes epidemiology: collaborative analysis of diagnostic criteria in Europe. Lancet 354:617-621

5. Hanefeld M, Koehler C, Fuecker K, Henkel E, Schaper F, Temelkova-Kurktschiev T (2003) Insulin secretion and insulin sensitivity pattern is different in isolated impaired glucose tolerance and impaired fasting glucose: the risk factor in impaired glucose tolerance for atherosclerosis and diabetes study. Diabetes Care 26:868-874

6. Tripathy D, Carlsson M, Almgren P et al (2000) Insulin secretion and insulin sensitivity in relation to glucose tolerance: lessons from the Botnia Study. Diabetes 49:975-980

7. Jensen CC, Cnop M, Hull RL, Fujimoto WY, Kahn SE (2002) Beta-cell function is a major contributor to oral glucose tolerance in high-risk relatives of four ethnic groups in the U.S. Diabetes 51:2170-2178

8. Carnevale Schianca GP, Rossi A, Sainaghi PP, Maduli E, Bartoli E (2003) The significance of impaired fasting glucose versus impaired glucose tolerance: importance of insulin secretion and resistance. Diabetes Care 26:1333-1337

9. Carlsson S, Persson PG, Alvarsson, M et al (1998) Weight history, glucose intolerance, and insulin levels in middle-aged Swedish men. Am J Epidemiol 148:539-545

10. Carlsson S, Persson PG, Alvarsson M et al (1999) Low birth weight, family history of diabetes, and glucose intolerance in Swedish middle-aged men. Diabetes Care 22:1043-1047

11. Grill V, Persson G, Carlsson S et al (1999) Family history of diabetes in middle-aged Swedish men is a gender unrelated factor which associates with insulinopenia in newly diagnosed diabetic subjects. Diabetologia 42:15-23

12. Agardh EE, Ahlbom A, Andersson T et al (2003) Work stress and low sense of coherence is associated with type 2 diabetes in middle-aged Swedish women. Diabetes Care 26:719-724

13. WHO (1985) Diabetes mellitus. Report of a WHO Study Group. WHO Tech Rep Ser 727:1-113

14. Expert Committee on the Diagnosis and Classification of Diabetes Mellitus (1997) Report of the expert committee on the diagnosis and classification of diabetes mellitus. Diabetes Care 20:1183-1197
15. Matthews DR, Hosker JP, Rudenski AS, Naylor BA, Treacher DF, Turner RC (1985) Homeostasis model assessment: insulin resistance and $\beta$-cell function from fasting plasma glucose and insulin concentrations in man. Diabetologia 28:412-419

16. Herbert V, Lau KS, Gottlieb CW, Bleicher SJ (1965) Coated charcoal immunoassay of insulin. J Clin Endocrinol Metab 25:1375-1384

17. Group DS (2003) Age- and sex-specific prevalences of diabetes and impaired glucose regulation in 13 European cohorts. Diabetes Care 26:61-69

18. Groop L, Forsblom C, Lehtovirta M et al (1996) Metabolic consequences of a family history of NIDDM (the Botnia study). Diabetes 45:1585-1593

19. Hanley AJ, D’Agostino R Jr, Wagenknecht LE et al (2002) Increased proinsulin levels and decreased acute insulin response independently predict the incidence of type 2 diabetes in the insulin resistance atherosclerosis study. Diabetes 51:12631270

20. Haffner SM, Mykkinän L, Valdez R et al (1994) Disproportionately increased proinsulin levels are associated with the insulin resistance syndrome. J Clin Endocrinol Metab 79:1806-1810

21. Zethelius B, Byberg L, Hales CN, Lithell H, Berne C (2002) Proinsulin is an independent predictor of coronary heart disease: report from a 27-year follow-up study. Circulation 105:2153-2158

22. Nagi DK, Hendra TJ, Ryle AJ et al (1990) The relationship of concentrations of insulin, intact proinsulin and 32-33 split proinsulin with cardiovascular risk factors in type 2 (noninsulin-dependent) diabetic subjects. Diabetologia 33:532-537

23. Lindahl B, Dinesen B, Eliasson M et al (1999) High proinsulin concentration precedes acute myocardial infarction in a nondiabetic population. Metabolism 48:1197-1202

24. Båvenholm P, Karpe F, Proudler A, Tornvall P, Crook D, Hamsten A (1995) Association of insulin and insulin propeptides with an atherogenic lipoprotein phenotype. Metabolism 44:1481-1488

25. DeFronzo RA, Ferrannini E, Simonson DC (1989) Fasting hyperglycemia in non-insulin-dependent diabetes mellitus: contributions of excessive hepatic glucose production and impaired tissue glucose uptake. Metabolism 38:387-395

26. Haffner SM, Miettinen H, Gaskill SP, Stern MP (1996) Decreased insulin action and insulin secretion predict the development of impaired glucose tolerance. Diabetologia 39:1201-1207

27. Båvenholm NP, Pigon J, Östenson C-G, Efendic S (2001) Insulin sensitivity of suppression of endogenous glucose production is the single most important determinator of glucose tolerance. Diabetes 50:1449-1454

28. Firth RG, Bell PM, Marsh HM, Hansen I, Rizza RA (1986) Postprandial hyperglycemia in patients with noninsulin-dependent diabetes mellitus. Role of hepatic and extrahepatic tissues. J Clin Invest 77:1525-1532

29. Ferrannini E, Bjorkman O, Reichard GAJ et al (1985) The disposal of an oral glucose load in healthy subjects. A quantitative study. Diabetes 34:580-588 